\title{
Efficacy of a Chinese Herbal Medicine Compound Zhangpi Ointment against Hydroxyurea-Induced Leg Ulcers: A Prospective, Randomized, Open-Label, Controlled Clinical Trial
}

\author{
Yu-yang Pang, ${ }^{1}$ Yan Li, ${ }^{2}$ Gang Kui, ${ }^{2}$ Yong Tang, ${ }^{1}$ Ming-juan Liao, ${ }^{2}$ Yong-ling Wang, \\ Zhen-dong Cao, ${ }^{2}$ and Qi Zhu ${ }^{1}{ }^{1}$ \\ ${ }^{1}$ Department of Hematology, Shanghai Ninth People’s Hospital, Shanghai Jiao Tong University School of Medicine, \\ Shanghai 200011, China \\ ${ }^{2}$ Department of Traditional Chinese Medicine, Shanghai Ninth People's Hospital, Shanghai Jiao Tong University School of Medicine, \\ Shanghai 200011, China
}

Correspondence should be addressed to Qi Zhu; zhuqi70@hotmail.com

Received 4 July 2018; Accepted 23 October 2018; Published 12 December 2018

Academic Editor: Yoshiyuki Kimura

Copyright (c) 2018 Yu-yang Pang et al. This is an open access article distributed under the Creative Commons Attribution License, which permits unrestricted use, distribution, and reproduction in any medium, provided the original work is properly cited.

Objective. The randomized controlled trial was to evaluate the efficacy of topical Chinese herbal Zhangpi Ointment for hydroxyureainduced leg ulcers in patients with myeloproliferative neoplasms. Patients and Methods. This single-center, prospective, randomized, open-label, controlled clinical trial conducted at Shanghai Ninth People's Hospital enrolled 54 patients with hydroxyurea-induced leg ulcers. Patients were randomly assigned to the control group $(n=27)$ treated with chlorhexidine dressing or the intervention group $(n=27)$ treated with the Zhangpi Ointment. Finally, 26 patients in the control group and 23 patients in the intervention group completed 8 weeks of observation. Results. The rate of complete healing was $100 \%$ for the intervention group, which was significantly higher than that of the control group $(96.15 \%)(P<0.05)$. Furthermore, the intervention group achieved a significantly higher rate of wound healing $(95.56 \%)$ than the control group $(69.02 \%)$ at week $4(P<0.01)$. The intervention group took $34 \pm 5$ days to achieve complete healing while the control group took $41 \pm 7$ days $(P<0.01)$. Moreover, grade $3 / 4$ side effects were observed in neither group. Conclusion. The Zhangpi Ointment is effective in promoting the healing of hydroxyurea-induced leg ulcers in patients with myeloproliferative neoplasms, providing a therapeutic option for a condition that is recalcitrant to conventional therapy.

\section{Introduction}

Myeloproliferative neoplasms (MPNs) are a group of stem cell disorders characterized by clonal myeloproliferation that is devoid of dyserythropoiesis, granulocytic dysplasia, or monocytosis. Hydroxyurea (HU) is an S-phase specific chemotherapeutic agent which inhibits DNA synthesis through its action on ribonucleotide diphosphate reductase [1] and is widely used in the treatment of MPNs. Although $\mathrm{HU}$ is relatively well tolerated, adverse effects often occur in $9 \%$ of treated patients $[2,3]$. Rare and severe adverse effects appear to be associated with long-term administration and may be systemic or restricted to skin and mucous membranes
[4]. The occurrence of painful leg ulcers represents another rare and incompletely characterized complication that has been described in patients with myeloproliferative diseases receiving high-dose (mean dose $28.2 \mathrm{mg} / \mathrm{kg}$ ) and longterm (mean treatment duration 39 months) HU treatment $[5,6]$. This cutaneous adverse effect is often treatmentresistant, causing delay in healing of the lesion (mean healing duration 4.3 months) [7]. Neovascularization and circulation deficiency and macroerythrocytosis during hydroxyurea treatment may be involved in the development of these rare ulcers, via impairment of the microcirculatory rheology [8]. Poor response to traditional local and systemic therapy is a typical feature of $\mathrm{HU}$-induced leg ulcers, and discontinuation 
of the drug is often required to achieve complete wound healing $[9,10]$. Although discontinuation of treatment is still the option of choice for complete recovery [11], leg ulcer can recur even after discontinuation of HU treatment [12]. Chlorhexidine diacetate released from wound dressing was used as an antimicrobic drug for the treatment of wound including skin ulcers [13]. It has been reported that two patients were successfully treated with a kind of matrix metalloproteinase modulator which is able to modulate the activity of proteases [14] and one patient was successfully treated with a topical basic fibroblast growth factor product, leading to rapid improvement within 2 weeks, without adverse effects [15]. However, the sample size was too small.

Zhangpi Ointment (ZPO) is a mixture of six topical Chinese herbs and two natural minerals, which include Glycyrrhiza uralensis Fisch, Angelica sinensis (Oliv.) Diels, Arnebia euchroma (Royle) Johnst., Rheum officinale Baill, Rehmannia glutinosa Libosch, Cortex Lycii, rubber powder, and calomel. Licoflavone, a component of Glycyrrhiza uralensis Fisch, was reported to promote gastric mucosal ulcer healing in mice by regulating inflammatory mediators [16]. Gastric ulcer healing effect has also been described for Angelica sinensis (Oliv.) Diels and other herbs [17, 18]. These findings, however, were mostly based on the effects of the medicinal herbs on gastrointestinal mucosal ulcers and in rodent models. No studies have been carried out on the effects of these medicinal herbs on leg ulcers and in humans.

Our anecdotal clinical experience suggests that the ZPO may be effective in promoting healing of $\mathrm{HU}$-induced leg ulcers. In the current prospective randomized controlled trial, we sought to investigate the efficacy of the ZPO for HUinduced leg ulcers in 54 patients with MPNs.

\section{Materials and Methods}

2.1. Trial Design and Participants. The study protocol was designed by experts from Department of Hematology and Traditional Chinese Medicine, Shanghai Ninth People's Hospital. Study approval was obtained from ethics committees at our hospital. They were responsible for the training and guidance of investigators and oversaw all aspects of coordination and data collection. Records of the allocation details were kept at our hospital for data verification and checking at monitoring visits. The study was undertaken in accordance with the Declaration of Helsinki and registered at http://www.chictr.org.cn (Clinical Trials. Gov ID: ChiCTR-INR-16009001).

This was a single-center, prospective, randomized, openlabel, controlled clinical trial of ZPO versus chlorhexidine dressing for HU-induced leg ulcers in patients with MPNs. We recruited 71 patients from Shanghai Ninth People's Hospital between July 2016 and April 2018. Eligible patients were aged at least 18 years and no older than 70 years, had histologically or cytologically confirmed MPNs which were treated with $\mathrm{HU}$, and had grade 1 to 3 leg ulcer (Wagner's classification) that had persisted for at least 2 months. Patients who had severe infection or autoimmune disease, severe liver, kidney, cardiovascular and mental diseases, diabetes, severe malnutrition, or history of bleeding disorder were excluded. Enrolled patients who had severe adverse events, who had poor compliance, and who asked for withdrawal were eliminated. All patients provided written informed consent.

The primary objective was to compare rate of complete healing with co-primary analyses of ulcer improvement (changes in the area of the ulcer) and healing time (number of days taken to achieve complete wound healing). Secondary endpoint was adverse events which were evaluated and graded according to Common Terminology Criteria for Adverse Events (NCICTCAE 4.0).

2.2. Procedures. Based on our preliminary experimental results, the rate of complete healing of the intervention group and control group at the fourth week was $95 \%$ and $64 \%$, respectively. Accordingly, in this superiority trial, the sample size was 54 (with $\alpha=0.05$, two-tailed and $\beta=0.2$ ). All eligible patients were randomly assigned on a 1:1 basis to receive either $\mathrm{ZPO}$ or chlorhexidine dressing with standard wound care. Randomization lists were generated through http://www.randomizer.org and held at our hospital.

The pharmacological properties of ZPO are shown in Table 1. ZPO was manufactured by the pharmacy of our hospital by the standard preparation method of traditional Chinese ointment medicine. The entire process was carried out in a bacteria-free environment and with strict quality control.

Standard wound care included local debridement of necrotic tissue or callus and dressing changes once daily until the wounds were completely healed [19]. All wounds were cleansed with sterile saline prior to assessment and dressing application. ZPO (about $1 \mathrm{~mm}$ thick) was applied to the surface of the ulcer which was then covered by a sterile dressing. For the control group, chlorhexidine dressing was applied to the surface of the ulcer directly. The duration of follow-up was 8 weeks and every patient was followed up once a week. Changes in the area of the ulcer $\left(\mathrm{cm}^{2}\right)$ were assessed at each follow-up visit.

2.3. Statistical Analysis. Changes in the area of the ulcer $\left(\mathrm{cm}^{2}\right)$ were measured by digital photography on day 0 and at each follow-up visit and calculated using the Image-Pro ه Plus 6.0 (Media Cybernetics, Inc., Bethesda, MD, USA ) analysis software. The rate of wound healing was calculated according to the following formula: area healed (\%) $=$ (initial area - the residual wound area) / initial area $\times 100 \%$. The rate of complete healing was defined as the percentage of patients who had complete $(100 \%)$ healing: complete healing $(\%)=$ number of patients who had complete healing / total number of patients $\times 100 \%$. Pathological scar formation was assessed according to the Vancouver Scar Scale [20].

The efficacy statistical analyses were prespecified and followed the intention-to-treat principle. All patients with a baseline assessment and at least one post-baseline assessment were included in analyses. Furthermore, patients who were randomized, received at least one treatment, and were assessed for adverse events were included in adverse events analysis. Continuous variables were described in mean \pm standard deviation and were compared between groups by 


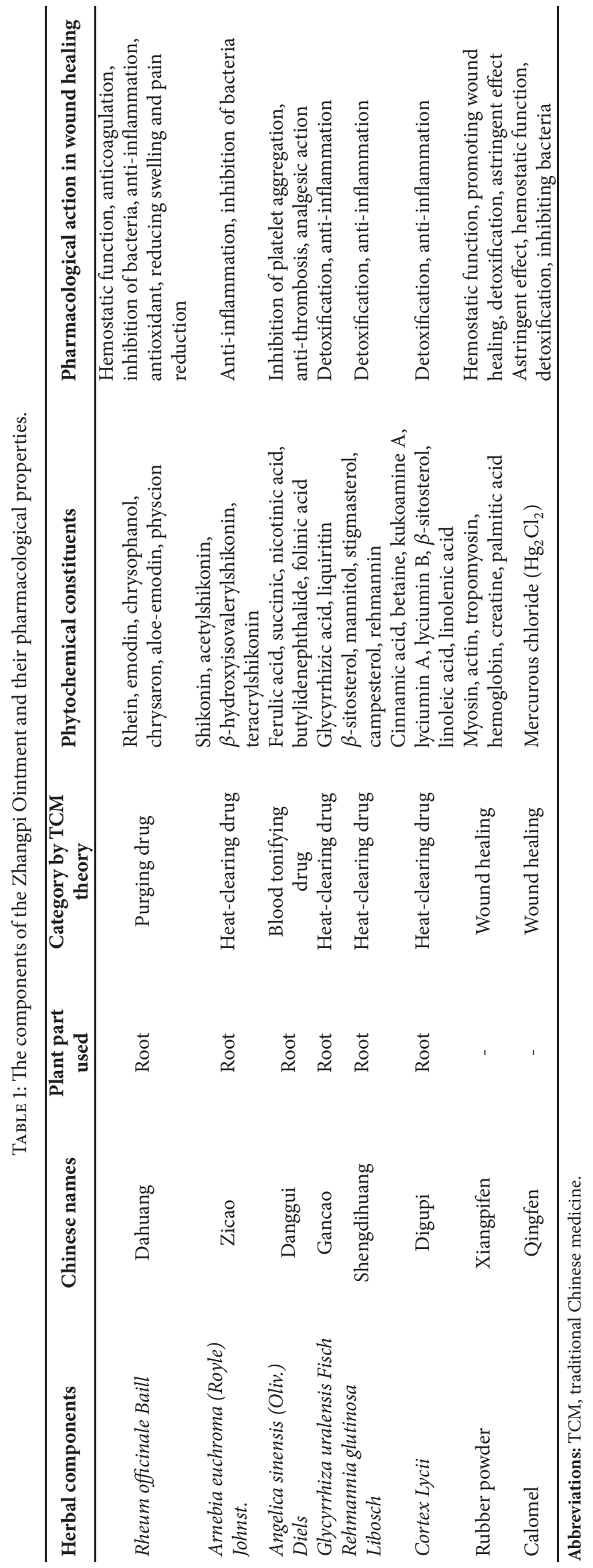




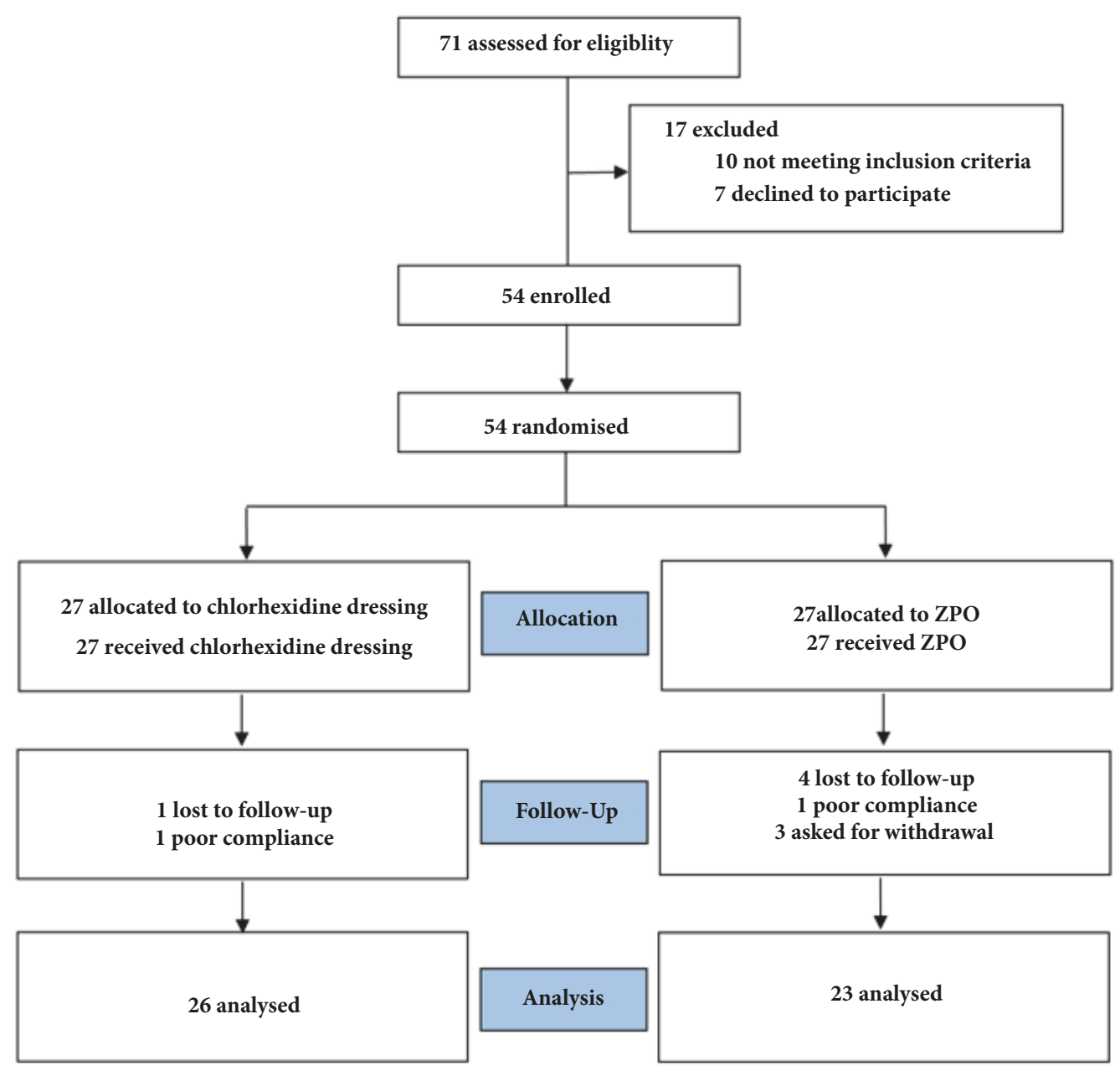

Abbreviations: ZPO, Zhangpi Ointment.

FIGURE 1: Flow of participants through the trial.

using the Wilcoxon rank sum test or rank ANCOVA for baseline adjustments. Student's $t$ test was used to compare proportions and also to test for heterogeneity within subgroups defined by baseline characteristics. A $P$ value $<0.05$ was considered statistically significant. All calculations were performed using SPSS 18.0 (SPSS Inc., Chicago, IL, USA).

\section{Results}

3.1. Participant Flow. The study flowchart is shown in Figure 1. Seventy-one patients who developed leg ulcers after undergoing HU therapy were recruited. Ten patients who did not meet the eligibility criteria and 7 patients who declined participation were excluded. Finally, 54 patients were enrolled in this clinical trial and were randomly assigned on a 1:1 basis to receive either ZPO or chlorhexidine dressing. Two patients lost to follow-up due to poor compliance and three patients asked for withdrawal. Consequently, 49 patients were analysed.

3.2. Patient Demographic and Baseline Characteristics. The demographic and baseline characteristics of the study population are shown in Table 2. The median age of the study population was 44 (range, 24 to 60 ) years and $61.2 \%$ of the patients were male. Thirty-five (71.4\%) patients were diagnosed with essential thrombocythemia, 9 (18.4\%) with polycythemia vera, and $5(10.2 \%)$ with myelofibrosis. Moreover, $59.2 \%$ of the patients had normal Body Mass Index (BMI). Each patient had one ulcer. The ulcers were evaluated according to the Wagner's classification and were classified to grade $1(26.5 \%)$, grade $2(42.9 \%)$, and grade $3(30.6 \%)$. Mean ulcer area was $3.5 \pm 1.0\left(\mathrm{~cm}^{2}\right)$. We conducted germiculture of the ulcers for all patients and $18.4 \%$ of the patients received systemic antibiotic therapy due to staphylococcus aureus infection. Meanwhile, most of the patients suffered moderate $(57.1 \%)$ and severe pain $(28.6 \%)$. All patients received standard doses of $\mathrm{HU}$ (15-20 mg/kg, once daily), which was terminated and replaced with recombined human interferon $\alpha(3,000,000$ units, three times a week) in 37 patients or watchful waiting in 12 patients when painful leg ulcers appeared. The median duration of ulcer prior to enrollment was 10 months. The two groups were comparable in demographic and baseline characteristics. 
TABLE 2: Baseline characteristics of the patients.

\begin{tabular}{|c|c|c|c|}
\hline Characteristic & Total $(n=49)$ & $\mathrm{ZPO}(\mathrm{n}=23)$ & Control $(n=26)$ \\
\hline Age (years), median and range & $44(24-60)$ & $45(24-60)$ & $44(29-60)$ \\
\hline \multicolumn{4}{|l|}{ Gender } \\
\hline Male & $30(61.2 \%)$ & $12(52.2 \%)$ & $18(69.2 \%)$ \\
\hline Female & $19(38.8 \%)$ & $11(47.8 \%)$ & $8(30.8 \%)$ \\
\hline \multicolumn{4}{|l|}{ Diagnosis } \\
\hline Essential thrombocythemia & $35(71.4 \%)$ & $16(69.6 \%)$ & $19(73.1 \%)$ \\
\hline Polycythemia vera & $9(18.4 \%)$ & $5(21.7 \%)$ & $4(15.4 \%)$ \\
\hline Myelofibrosis & $5(10.2 \%)$ & $2(8.7 \%)$ & $3(11.5 \%)$ \\
\hline Duration of ulcer at enrolment (months), median and range & $10(2-36)$ & $9(2-18)$ & $11(2-36)$ \\
\hline \multicolumn{4}{|l|}{ BMI $\left(\mathrm{kg} / \mathrm{m}^{2}\right)$} \\
\hline$<18.5$ & $12(24.5 \%)$ & $4(17.4 \%)$ & $8(30.8 \%)$ \\
\hline $18.5-24.9$ & $29(59.2 \%)$ & $16(69.6 \%)$ & $13(50.0 \%)$ \\
\hline$\geq 25$ & $8(16.3 \%)$ & $3(13.0 \%)$ & $5(19.2 \%)$ \\
\hline \multicolumn{4}{|l|}{ Treatment after withdrawal of $\mathrm{HU}$} \\
\hline Recombined human interferon $\alpha$ & $37(75.5 \%)$ & $16(69.6 \%)$ & $21(80.8 \%)$ \\
\hline Watchful waiting & $12(24.5 \%)$ & $7(30.4 \%)$ & $5(19.2 \%)$ \\
\hline \multicolumn{4}{|l|}{ Routine blood test } \\
\hline Leukocyte $\left(\times 10^{\wedge} 9 / \mathrm{L}\right)$ & $7.6 \pm 5.2$ & $8.8 \pm 5.4$ & $6.6 \pm 4.8$ \\
\hline Hemoglobin $(\mathrm{g} / \mathrm{L})$ & $115 \pm 36.3$ & $116 \pm 36.9$ & $114 \pm 37$ \\
\hline Platelet $\left(\times 10^{\wedge} 9 / \mathrm{L}\right)$ & $303 \pm 179$ & $298 \pm 167$ & $308 \pm 183$ \\
\hline Ulcer area $(\mathrm{cm} 2)$ & $3.5 \pm 1.0$ & $3.3 \pm 1.0$ & $3.6 \pm 1.0$ \\
\hline \multicolumn{4}{|l|}{ Wagner's classification of the ulcers } \\
\hline Grade 1 & $13(26.5 \%)$ & $5(21.7 \%)$ & $8(30.8 \%)$ \\
\hline Grade 2 & $21(42.9 \%)$ & $9(39.1 \%)$ & $12(46.1 \%)$ \\
\hline Grade 3 & $15(30.6 \%)$ & $9(39.1 \%)$ & $6(23.1 \%)$ \\
\hline \multicolumn{4}{|l|}{ Germiculture of the ulcers } \\
\hline Positive & $9(18.4 \%)$ & $6(26.1 \%)$ & $3(11.5 \%)$ \\
\hline Negative & $40(81.6 \%)$ & $17(73.9 \%)$ & $23(88.5 \%)$ \\
\hline \multicolumn{4}{|l|}{ Pain evaluation (NRS classification) } \\
\hline $0-3$ & $7(14.3 \%)$ & $3(13.0 \%)$ & $4(15.4 \%)$ \\
\hline $4-6$ & $28(57.1 \%)$ & $15(65.3 \%)$ & $13(50.0 \%)$ \\
\hline $7-10$ & $14(28.6 \%)$ & $5(21.7 \%)$ & $9(34.6 \%)$ \\
\hline
\end{tabular}

Abbreviations: ZPO, Zhangpi Ointment; BMI, Body Mass Index; HU, hydroxyurea; NRS, Numerical Rating Scale.

3.3. Outcomes and Estimation. Forty-nine patients completed 8 weeks of treatment. The rate of complete healing was $100 \%$ for the intervention group, which was significantly higher than that of the control group $(96.15 \%)(P<0.05)$. Statistically significant difference in the rate of complete healing between the intervention group and the control group started to emerge at week 4 of treatment (intervention: $26.09 \%$ versus control: $6.90 \% ; P<0.05$ ) (Figure 2). Furthermore, the intervention group achieved a significantly higher rate of wound healing $(95.56 \%)$ than the control group $(69.02 \%)$ at week $4(P<0.01)$ (Figure 3$)$. The intervention group took 34 \pm 5 days to achieve complete healing while the control group took $41 \pm 7$ days $(P<0.01)$ (Figure 4$)$. Moreover, the number of pathological scars was significantly smaller in the intervention group than the control group $(P<0.01)$ (Figure 5).

3.4. Adverse Events. Adverse events were evaluated and graded according to Common Terminology Criteria for

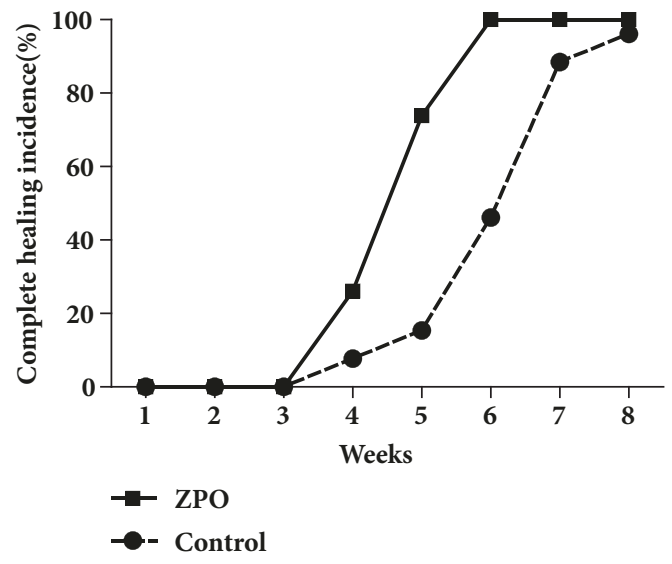

Abbreviations: ZPO, Zhangpi Ointment.

FIGURE 2: Complete healing incidence during the overall observation period of eight weeks. 

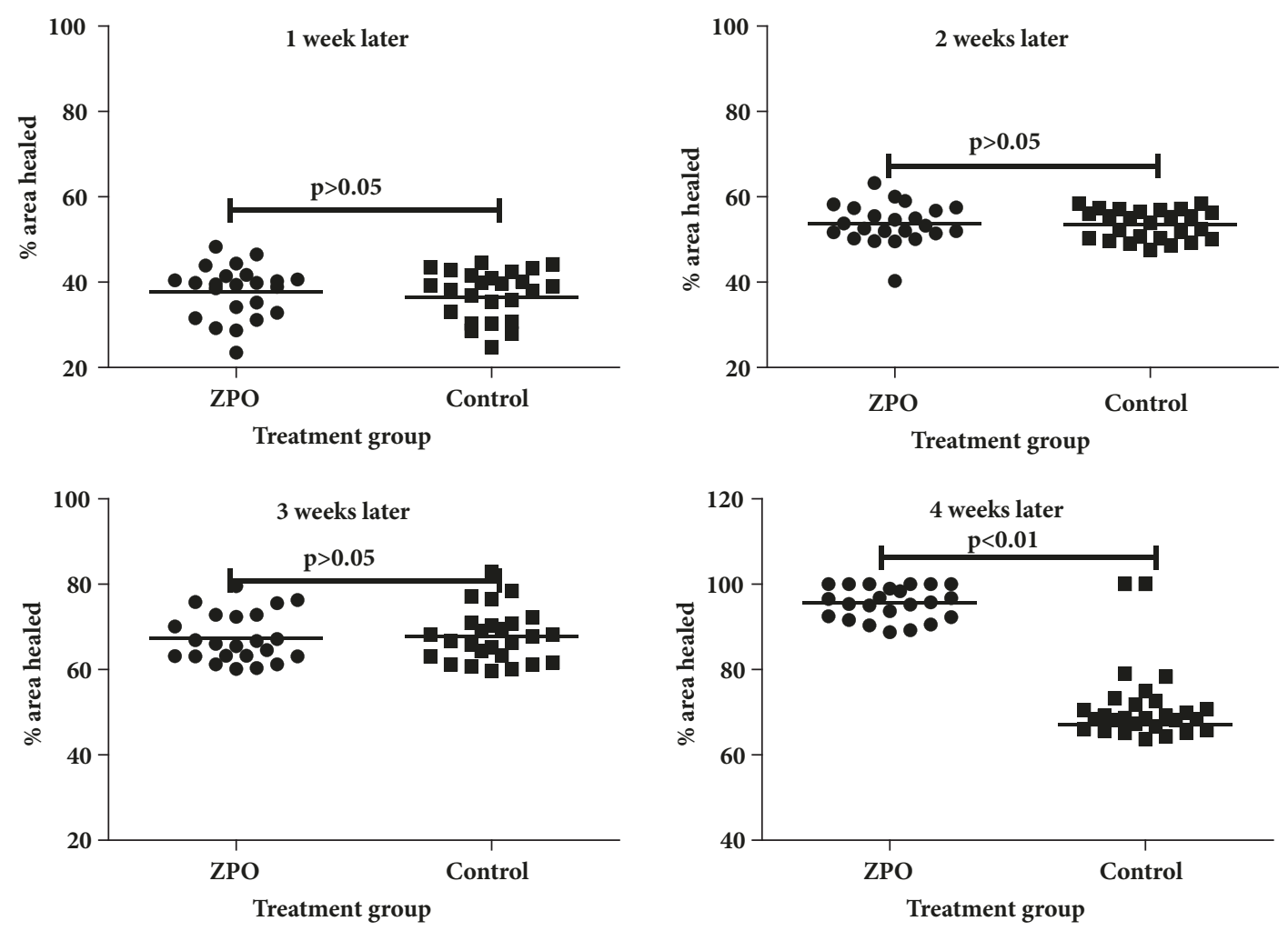

Abbreviations: ZPO, Zhangpi Ointment.

Figure 3: Mean \% of ulcer area healed at each time point of assessment.

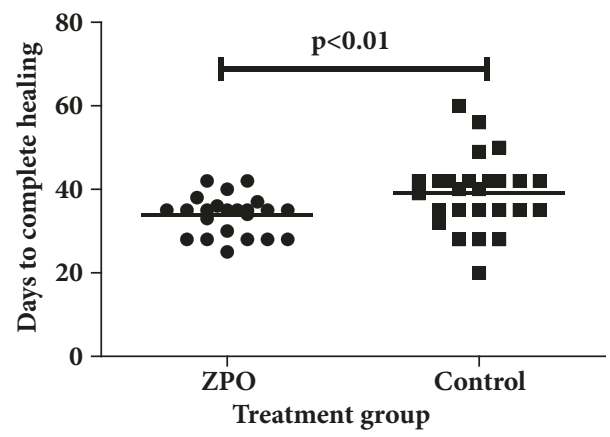

Abbreviations: ZPO, Zhangpi Ointment.

FIGURE 4: Days to complete ulcer healing of the two groups.

Adverse Events (NCICTCAE 4.0). Table 3 shows the frequency of common side effects. Grade 3/4 side effects were observed in neither group, and no patients withdrew due to side effects. Grade 2 dry skin and skin tenderness, and grade 1 liver disorder were seen in 1 patient each in the intervention group. Grade 2 liver disorder was seen in 1 patient in the control group.

\section{Discussion}

HU-induced leg ulcers, though rare, are recalcitrant to treatment and mandate cessation of HU therapy [21]. The

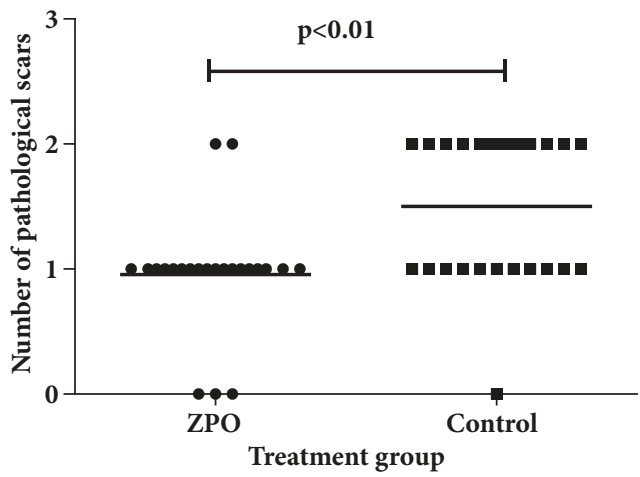

Abbreviations: ZPO, Zhangpi Ointment.

FIgURE 5: Pathological scar formation after complete healing for the two groups.

present randomized trial showed that the Chinese medicinal herbal ZPO was effective in promoting the healing of HUinduced leg ulcers, providing an alternative treatment for the disease.

HU remains the therapeutic choice for myeloproliferative neoplasms such as polycythemia vera [19], essential thrombocythemia [22], and myelofibrosis [23]. Painful leg ulcers are a rare but troublesome complication that has been described in patients receiving high-dose, long-term HU treatment, 
TABLE 3: Adverse events in the study population.

\begin{tabular}{|c|c|c|c|c|c|c|c|c|}
\hline & \multicolumn{4}{|c|}{ Intervention (\%) } & \multicolumn{4}{|c|}{ Control (\%) } \\
\hline & Grade 1 & Grade 2 & Grade 3 & Grade 4 & Grade 1 & Grade 2 & Grade 3 & Grade 4 \\
\hline \multicolumn{9}{|l|}{ Cutaneous } \\
\hline Allergy & $0(0.00)$ & $0(0.00)$ & $0(0.00)$ & $0(0.00)$ & $0(0.00)$ & $0(0.00)$ & $0(0.00)$ & $0(0.00)$ \\
\hline Infections & $0(0.00)$ & $0(0.00)$ & $0(0.00)$ & $0(0.00)$ & $1(3.85)$ & $0(0.00)$ & $0(0.00)$ & $0(0.00)$ \\
\hline Bleeding & $1(4.35)$ & $0(0.00)$ & $0(0.00)$ & $0(0.00)$ & $1(3.85)$ & $1(3.85)$ & $0(0.00)$ & $0(0.00)$ \\
\hline Dry skin & $0(0.00)$ & $1(4.35)$ & $0(0.00)$ & $0(0.00)$ & $1(3.85)$ & $0(0.00)$ & $0(0.00)$ & $0(0.00)$ \\
\hline Skin tenderness & $1(4.35)$ & $1(4.35)$ & $0(0.00)$ & $0(0.00)$ & $2(7.69)$ & $0(0.00)$ & $0(0.00)$ & $0(0.00)$ \\
\hline \multicolumn{9}{|l|}{ Non-cutaneous } \\
\hline Renal disorders & $0(0.00)$ & $0(0.00)$ & $0(0.00)$ & $0(0.00)$ & $0(0.00)$ & $0(0.00)$ & $0(0.00)$ & $0(0.00)$ \\
\hline Liver disorders & $1(4.35)$ & $0(0.00)$ & $0(0.00)$ & $0(0.00)$ & $0(0.00)$ & $1(3.85)$ & $0(0.00)$ & $0(0.00)$ \\
\hline
\end{tabular}

often leading to discontinuation of HU. Although the exact mechanism for the development of this complication is still unclear, it has been hypothesized that inadvertent minor injury to the malleolar region that is inadequately repaired may jeopardize the regenerative potential of the epidermis. To date, only single case reports $[14,21,24,25]$ or singlecenter experiences [2, 26, 27] have been published regarding this phenomenon, with a reported incidence ranging from $5 \%$ to $10 \%$ of HU treated patients. A $5 \%$ incidence of skin ulcers during $\mathrm{HU}$ treatment also has been reported in a large randomized trial in patients with essential thrombocythemia [28]. Currently, the occurrence of skin ulcers during $\mathrm{HU}$ treatment is considered as HU intolerance [29].

Our data suggested that during the first three weeks, the two groups showed no statistically significant difference in the area healed (\%), but by week 4, patients receiving ZPO exhibited a significant improvement compared to the control group. Moreover, all patients in the intervention group achieved complete healing at week 4. In addition, the intervention group achieved healing significantly more rapidly than the control group, with significantly fewer pathological scars, highlighting the efficacy of ZPO in healing HU-induced leg ulcers.

The underlying mechanism for the ulcer healing effects of ZPO still remains unclear. In chronic skin ulcer, intense and persistent inflammatory response, reduced angiogenesis and cell proliferation, decreased cell growth factors and collagen synthesis and degradation of excessive extracellular matrix all contribute to impairment of wound healing. Amongst these, the regeneration of capillary and vascular network formation plays an important role. As the most important regulatory factor of angiogenesis and neovascularization, vascular endothelial growth factor (VEGF) can stimulate the formation, proliferation, and migration of endothelial cells of the blood vessels [30]. The continuous secretion of VEGF plays a very important role in accelerating wound healing. Another study conducted by our team on the treatment of nonhealing wounds after breast cancer operation showed that the expression of VEGF in wound tissues was upregulated in patients treated with ZPO (data not shown). Glycyrrhiza uralensis Fisch, a component of ZPO, was reported to promote gastric ulcer healing by regulating inflammatory mediators [16]. We speculate that the ZPO may improve wound healing via multiple mechanisms including modulating the content of VEGF or inflammatory mediators. The levels of these molecules were not investigated in the current study and are worthy of further exploration.

Given the limited treatment options for HU-induced leg ulcers, our current randomized controlled trial demonstrated that the ZPO offers an effective therapeutic option for HUinduced leg ulcers. The study has certain limitations. The study population is relatively small, given the rarity of the condition. In addition, our hospital is a tertiary care center and the findings of the study may not be applicable to primary care settings. Furthermore, a mixture of medicinal herbs was used in the ZPO, rendering it difficult to attribute the actions of the ointment to any particular compound.

In conclusion, our randomized controlled trial demonstrates that the ZPO is effective in promoting the healing of HU-induced leg ulcers in patients with myeloproliferative neoplasms, providing a therapeutic option for a condition that is recalcitrant to conventional therapy.

\section{Abbreviations}

MPNs: Myeloproliferative neoplasms

HU: Hydroxyurea

ZPO: Zhangpi Ointment

VEGF: Vascular endothelial growth factor.

\section{Data Availability}

The data used to support the findings of this study are available from the corresponding author upon request.

\section{Conflicts of Interest}

All authors have no conflicts of interest to declare.

\section{Authors' Contributions}

Yu-yang Pang, Yan Li, Gang Kui, and Yong Tang contributed equally to this work.

\section{Acknowledgments}

This work was supported in part by grants from Integration Fund and Fund of Shanghai Ninth People's Hospital affiliated to Shanghai Jiao Tong University School of Medicine (grant 
No. 2014B02), Shanghai Committee of Science and Technology (grant No. 12ZR1416800, 13ZR1423800), and National Natural Science Foundation of China (grant No. 81870156). The authors are grateful to all the members from the Hematology and Traditional Chinese Medicine Department of Shanghai Ninth People's Hospital for their continuous support and encouragement.

\section{References}

[1] J. W. Yarbro, "Further Studies on the Mechanism of Action of Hydroxyurea," Cancer Research, vol. 28, no. 6, pp. 1082-1087, 1968.

[2] Y. Najean and J.-D. Rain, "Treatment of polycythemia vera: The use of hydroxyurea and pipobroman in 292 patients under the age of 65 years," Blood, vol. 90, no. 9, pp. 3370-3377, 1997.

[3] J. R. Mekkes, M. A. M. Loots, A. C. Van Der Wal, and J. D. Bos, "Causes, investigation and treatment of leg ulceration," British Journal of Dermatology, vol. 148, no. 3, pp. 388-401, 2003.

[4] E. Antonioli, P. Guglielmelli, L. Pieri et al., "Hydroxyurearelated toxicity in 3,411 patients with Ph'-negative MPN," American Journal of Hematology, vol. 87, no. 5, pp. 552-554, 2012.

[5] R. L. Stahl and R. Silber, "Vasculitic leg ulcers in chronic myelogenous leukemia," American Journal of Medicine, vol. 78, no. 5, pp. 869-872, 1985.

[6] A. Antar, R. S. Ishak, Z. K. Otrock et al., "Successful treatment of hydroxyurea-associated chronic leg ulcers associated with squamous cell carcinoma," Hematology/Oncology and Stem Cell Therapy, vol. 7, no. 4, pp. 166-169, 2014.

[7] E. Montefusco, G. Alimena, R. Gastaldi, O. A. Carlesimo, G. Valesini, and F. Mandelli, "Unusual dermatologic toxicity of long-term therapy with hydroxyurea in chronic myelogenous leukemia," TUMORI, vol. 72, no. 3, pp. 317-321, 1986.

[8] S. Tsuchiya, S. Ichioka, and N. Sekiya, "Hydroxyurea-induced foot ulcer in a case of essential thrombocythaemia," Journal of Wound Care, vol. 19, no. 8, pp. 361-364, 2010.

[9] U. Bader, M. Banyai, R. Böni, G. Burg, and J. Hafner, "Leg ulcers in patients with myeloproliferative disorders: Diseaseor treatment-related?" Dermatology, vol. 200, no. 1, pp. 45-48, 2000.

[10] G. Weinlich, G. Schuler, R. Greil, H. Kofler, and P. Fritsch, "Leg ulcers associated with long-term hydroxyurea therapy," Journal of the American Academy of Dermatology, vol. 39, no. 2, pp. 372374, 1998.

[11] A. Khaled, N. El Guellali, L. Aïssaoui et al., "Hydroxyurea induced-Leg ulcer in polycythemia vera," La Tunisie Médicale, vol. 89, no. 3, pp. 292-294, 2011.

[12] K. Kikuchi, K. Arita, Y. Tateishi, M. Onozawa, M. Akiyama, and H. Shimizu, "Recurrence of hydroxyurea-induced leg ulcer after discontinuation of treatment," Acta Dermato-Venereologica, vol. 91, no. 3, pp. 373-374, 2011.

[13] S. Rossi, M. Marciello, G. Sandri et al., "Wound dressings based on chitosans and hyaluronic acid for the release of chlorhexidine diacetate in skin ulcer therapy," Pharmaceutical Development and Technology, vol. 12, no. 4, pp. 415-422, 2007.

[14] M. Romanelli, V. Dini, and P. Romanelli, "Hydroxyureainduced leg ulcers treated with a protease-modulating matrix," JAMA Dermatology, vol. 143, no. 10, pp. 1310-1313, 2007.

[15] Y. Aragane, T. Okamoto, A. Yajima, R. Isogai, A. Kawada, and T. Tezuka, "Hydroxyurea-induced foot ulcer successfully treated with a topical basic fibroblast growth factor product," British Journal of Dermatology, vol. 148, no. 3, pp. 599-600, 2003.

[16] Y. Yang, S. Wang, Y.-R. Bao et al., "Anti-ulcer effect and potential mechanism of licoflavone by regulating inflammation mediators and amino acid metabolism," Journal of Ethnopharmacology, vol. 199, pp. 175-182, 2017.

[17] Y. N. Ye, H. L. So, E. S. L. Liu, V. Y. Shin, and C. H. Cho, "Effect of polysaccharides from Angelica sinensis on gastric ulcer healing," Life Sciences, vol. 72, no. 8, pp. 925-932, 2003.

[18] H. Chen, O. J. Olatunji, and Y. Zhou, "Anti-oxidative, antisecretory and anti-inflammatory activities of the extract from the root bark of Lycium chinense (Cortex Lycii) against gastric ulcer in mice," Journal of Natural Medicines, vol. 70, no. 3, pp. 610-619, 2016.

[19] M. Eneroth and W. H. van Houtum, "The value of debridement and Vacuum-Assisted Closure (V.A.C.) Therapy in diabetic foot ulcers," Diabetes/Metabolism Research and Reviews, vol. 24, no. 1, pp. S76-S80, 2008.

[20] Z. Tyack, M. Simons, A. Spinks, and J. Wasiak, "A systematic review of the quality of burn scar rating scales for clinical and research use," Burns, vol. 38, no. 1, pp. 6-18, 2012.

[21] F. Stagno, P. Guglielmo, U. Consoli, P. Fiumara, M. Russo, and R. Giustolisi, "Successful healing of hydroxyurea-related leg ulcers with topical granulocyte-macrophage colony-stimulating factor," Blood, vol. 94, no. 4, pp. 1479-1480, 1999.

[22] T. Barbui, G. Barosi, A. Grossi et al., "Practice guidelines for the therapy of essential thrombocythemia. A statement from the Italian Society of Hematology, the Italian Society of Experimental Hematology and the Italian Group for Bone Marrow Transplantation," Haematologica, vol. 89, no. 2, pp. 215232, 2004.

[23] R. A. Mesa, "How I treat symptomatic splenomegaly in patients with myelofibrosis," Blood, vol. 113, no. 22, pp. 5394-5400, 2009.

[24] J. L. Sastre, A. Bravo, S. Tembrás, R. Gómez, and C. Ulibarrena, "Leg ulcers associated with hydroxyurea therapy.", Haematologica, vol. 88, no. 2, p. EIM01, 2003.

[25] S.-W. Hwang, S.-K. Hong, S.-H. Kim, J.-K. Seo, D. Lee, and H.-S. Sung, "A hydroxyurea-induced Leg ulcer," Annals of Dermatology, vol. 21, no. 1, pp. 39-41, 2009.

[26] M. L. Randi, E. Ruzzon, G. Luzzatto, F. Tezza, A. Girolami, and F. Fabris, "Safety profile of hydroxyurea in the treatment of patients with Philadelphia-negative chronic myeloproliferative disorders," Haematologica, vol. 90, no. 2, pp. 261-262, 2005.

[27] E. Ruzzon, M. L. Randi, F. Tezza, G. Luzzatto, R. Scandellari, and F. Fabris, "Leg ulcers in elderly on hydroxyurea: A single center experience in ph-myeloproliferative disorders and review of literature," Aging Clinical and Experimental Research, vol. 18, no. 3, pp. 187-190, 2006.

[28] C. N. Harrison, P. J. Campbell, G. Buck et al., "Hydroxyurea compared with anagrelide in high-risk essential thrombocythemia," The New England Journal of Medicine, vol. 353, no. 1, pp. 33-45, 2005.

[29] G. Barosi, G. Birgegard, G. Finazzi et al., "A unified definition of clinical resistance and intolerance to hydroxycarbamide in polycythaemia vera and primary myelofibrosis: Results of a European LeukemiaNet (ELN) consensus process," British Journal of Haematology, vol. 148, no. 6, pp. 961-963, 2010.

[30] J. L. Spivak and H. Hasselbalch, "Hydroxycarbamide: a user's guide for chronic myeloproliferative disorders," Expert Review of Anticancer Therapy, vol. 11, no. 3, pp. 403-414, 2011. 


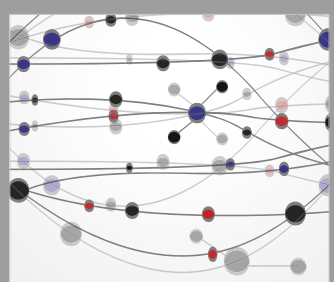

The Scientific World Journal
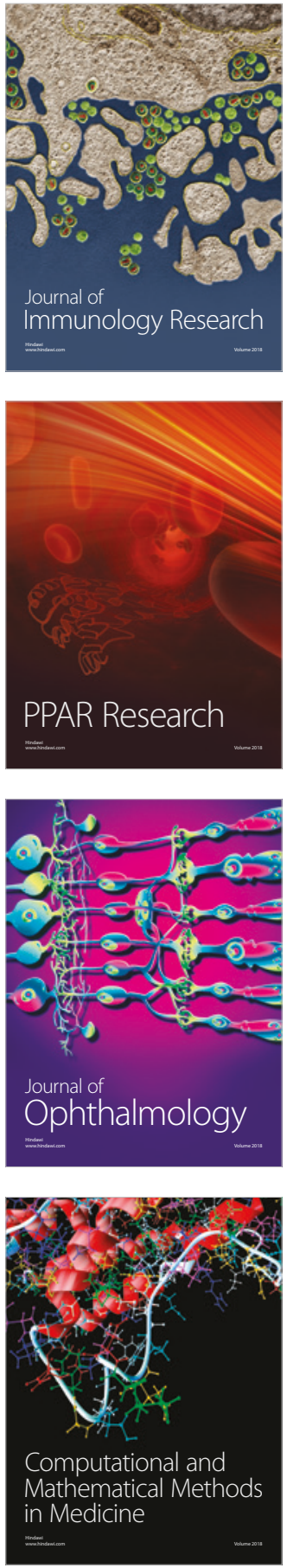

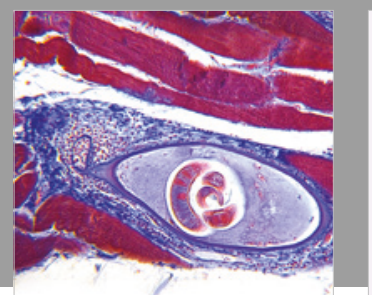

Gastroenterology Research and Practice

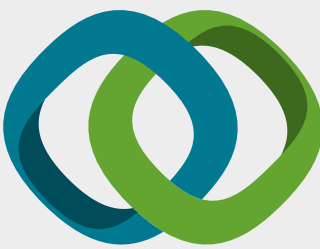

\section{Hindawi}

Submit your manuscripts at

www.hindawi.com
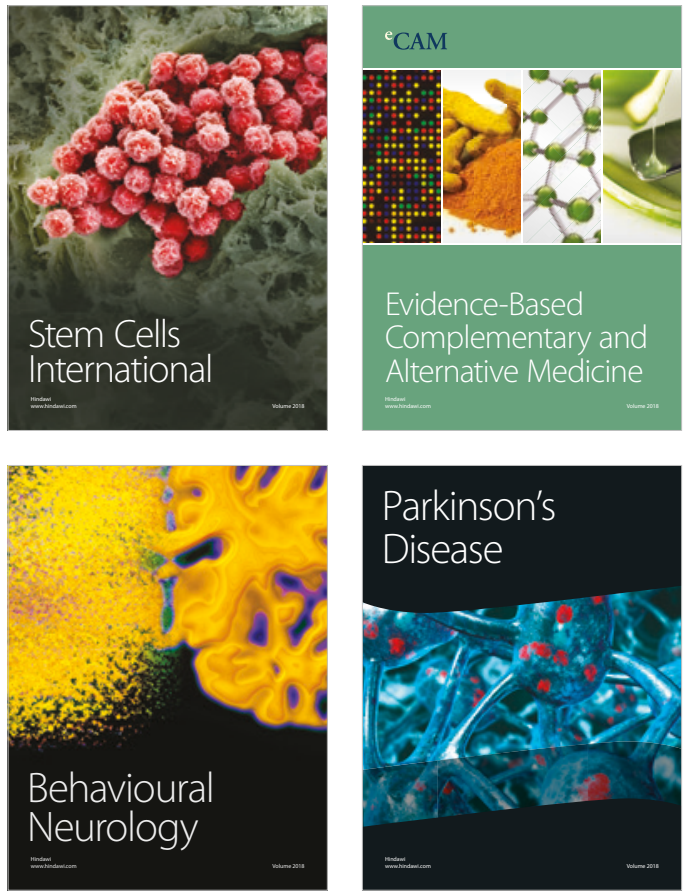

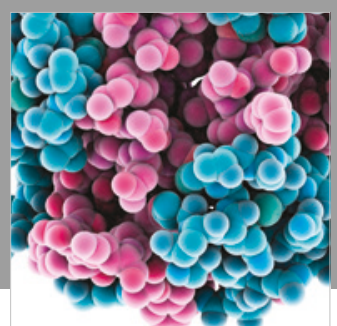

ournal of

Diabetes Research

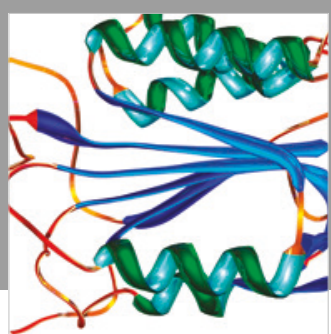

Disease Markers
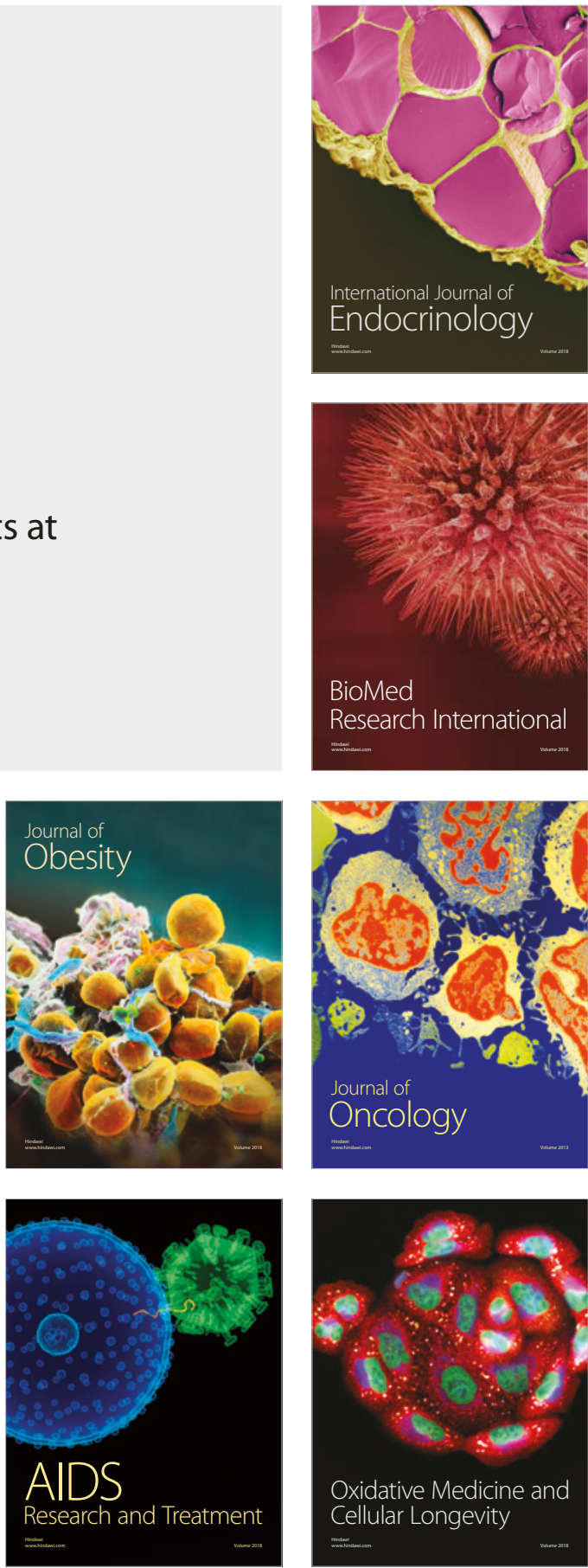